\title{
Manipulation of the swirling flow instability in hydraulic turbine diffuser by different methods of water injection
}

\author{
Pavel Rudolf ${ }^{1, *}$, Jiři Litera ${ }^{1}$, Germán Alejandro Ibarra Bolanos ${ }^{2}$ and David Štefan ${ }^{1}$ \\ ${ }^{1}$ V. Kaplan Dept. of Fluid Engineering, Faculty of Mechanical Engineering, Brno University of Technology, Technická 2896/2, Brno \\ 61669, Czech Republic \\ ${ }^{2}$ Research Group in Fluid Dynamics, School of Mechanical Engineering, Universidad del Valle, Calle 13 No. 100-00, Cali, Colombia
}

\begin{abstract}
Vortex rope, which induces substantial pressure pulsations, arises in the draft tube (diffuser) of Francis turbine for off-design operating conditions. Present paper focuses on mitigation of those pulsations using active water jet injection control. Several modifications of the original Susan-Resiga's idea were proposed. All modifications are driven by manipulation of the shear layer region, which is believed to play important role in swirling flow instability. While some of the methods provide results close to the original one, none of them works in such a wide range. Series of numerical experiments support the idea that the necessary condition for vortex rope pulsation mitigation is increasing the fluid momentum along the draft tube axis.
\end{abstract}

\section{Introduction}

Both financial subsidies and technological advancement enabled wide spread of renewable energy sources, especially wind and photovoltaic power plants. Increasing share of these sources, which are highly dependent on meteorology conditions or daylight raises concern about stability of the electrical grid. Grid control can be realized by such devices that have both sufficient power and short response time. These criteria are met especially in hydro power plants. Their role in the grid control increases and providing of so called ancillary services becomes even more important nowadays. Hydro power plants offer all three types of grid control (primary, secondary, tertiary), but also voltage support or compensation of active power loss with response time within tens of seconds. Another advantage is black start and island operation capability.

Most (more than $60 \%$ ) of hydro power plants with installed power bigger than $10 \mathrm{MW}$ is equipped with Francis turbines, moreover pump turbines (reversible Francis turbines) installed in pump storage power plants make up almost another $10 \%$ of all hydraulic turbines worldwide.

\section{Vortex rope in hydraulic turbine diffuser}

Requirements on the control ability of hydro power plants enforce their operation in off-design conditions (deep part load, part load, upper part load, overload). However single controlled hydraulic turbines (e.g. Francis turbine) impose highly swirling flow on the turbine runner exit. The swirl can be explained by mismatch of moment of momentum produced by guide vanes and moment of momentum consumed by the runner. The residual swirl leaves from the runner to turbine draft tube in form of circumferential velocity $v_{u}$ (see Fig.1). Draft tube is a diffuser, which converts dynamic pressure into static pressure and thereby increases turbine efficiency. Swirling flow is very susceptible to instabilities. For case of the part load the instability is in form of spiral vortex, which is a result of so called spiral vortex breakdown (see Fig.2). This strong coherent vortical structure is well known also in swirl burners or flow over the airplanes. Vortex breakdown is usually defined as sudden change of the flow structure at critical swirl level and is characterized by flow deceleration in vicinity of the axis, formation of stagnation point, onset of recirculation bubble and eventually vortices are rolled-up ( $\mathrm{KH}$ instability) along the shear layer in-between the two opposing streams. It means that the resulting vortex rope is wrapped around the low velocity zone, Fig.3.

a)

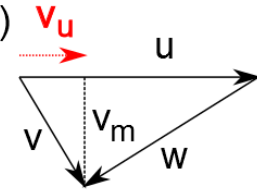

b)

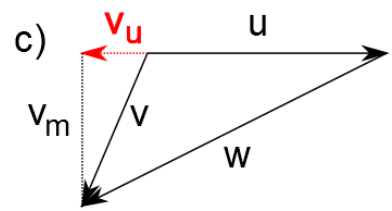

Fig. 1. Connection between the velocity triangle on runner exit and the residual swirl for a) partload, b) best efficiency point, c) overload

* Corresponding author: rudolf@fme.vutbr.cz 

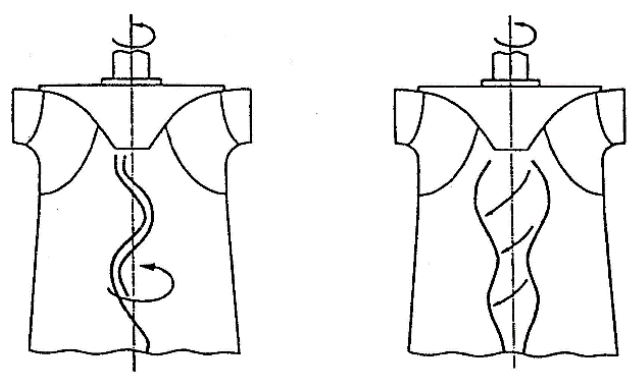

Fig. 2. Vortex rope in case of partload (left) and overload (right) turbine operation

The corkscrew vortex in turbine diffuser is also called a precessing vortex core (PVC) due to its kinematics [1, 2].

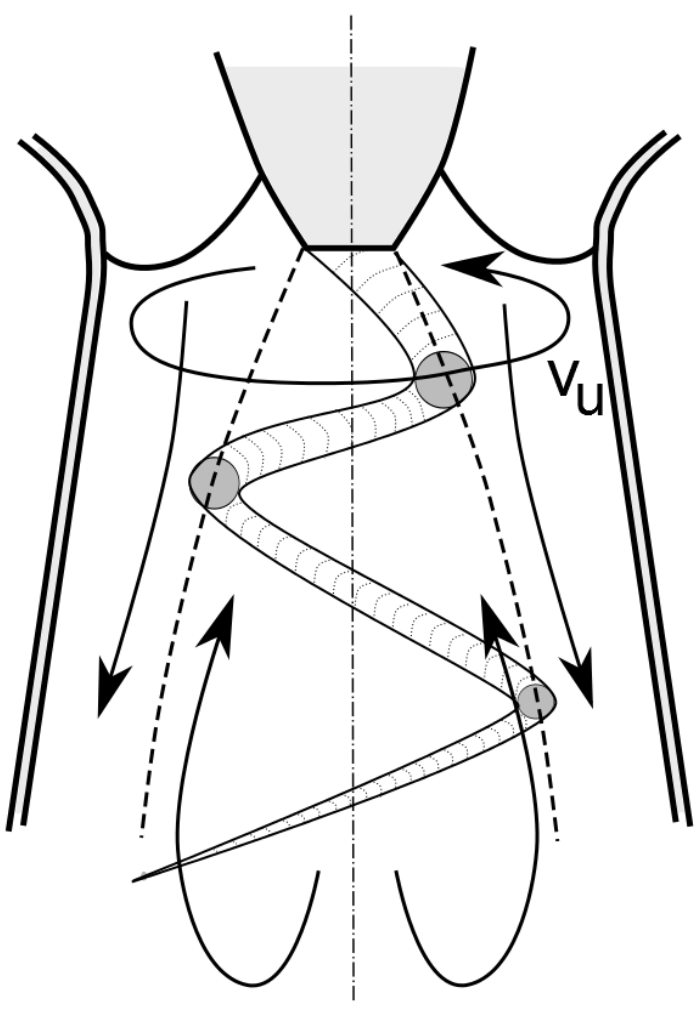

Fig. 3. Spiral vortex breakdown (dashed line marks the shear layer)

Precessing motion of the vortex rope is a source of pressure pulsations both in radial and axial direction. These pulsations can reach up to $5-10 \%$ of the turbine head and are manifested by vibrations and noise. Pulsations can also be transmitted upstream and cause runner vibrations. From point of dynamical description this type of pulsations belongs to low frequency ones, because their typical frequency is about one fifth to one fourth of the runner rotational frequency.

\section{Vortex rope control}

\subsection{Active water jet control designed by Politehnica Timisoara}

Since vortex rope is very dominant and strong swirling structure its mitigation is rather difficult. In practice usually air injection through runner cone is applied. However admission of air into the diffuser may cause additional dynamical problems related to increased compliance. Another way for vortex rope suppression is mounting the fins on draft tube walls, which decelerates the rotating flow, but also increases hydraulic losses.

Susan-Resiga et al put forward the idea of continuous water injection through runner cone in 1996 [3]. A complex test apparatus was built at Politehnica Timisoara to test the water injection in terms of pressure amplitudes suppression and the necessary water discharge [4], see Fig.4. Test apparatus uses a swirl generator with free runner to imitate flow field corresponding to partial load Francis turbine operation. Smart and elaborate idea of collecting the water for injection from downstream end of the draft tube was employed (so called flow feedback). This method takes advantage of the adverse pressure gradient in turbine diffuser.

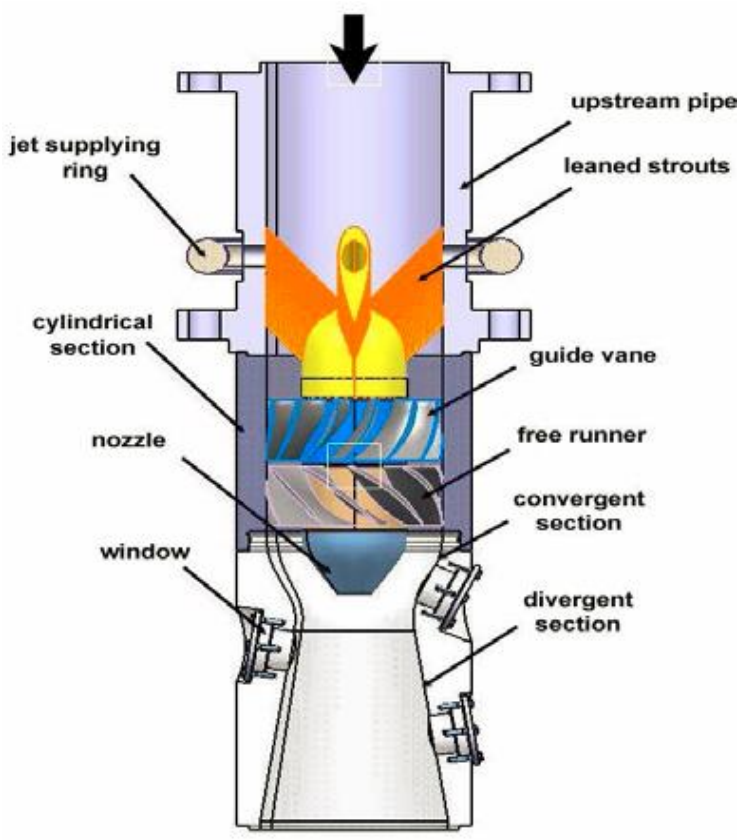

Fig. 4. Test apparatus at Politehnica Timisoara with free runner, plexiglass draft tube and set of pressure probes [4]

Static pressure probes were mounted in four locations on the draft tube wall (Fig. 5). Extensive testing proved viability of the proposed method at least in the laboratory conditions. However it was found that the discharge supplied by the adverse pressure gradient is not sufficient to provide full vortex rope elimination and additional discharge by external pump has to be employed. It means that the overall discharge for water injection is relatively high and potentially there might be more suitable locations were to apply this flow control. In terms static 
pressure amplitude mitigation, energy and discharge consumption.
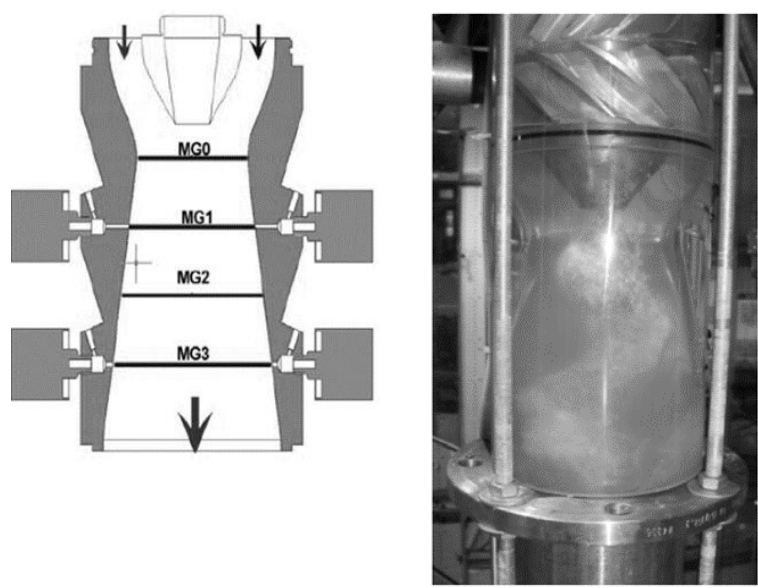

Fig. 5. Locations of pressure probes (left) and visualization of the vortex rope (right) [5]

\subsection{Computational modelling of the water injection and shear layer instability}

Commercial CFD code ANSYS Fluent was used for computational simulations of the flow downstream of the swirl generator. Boundary conditions for velocity and turbulence were adopted from [5]. Computational domain consisted of about half a million hexagonal elements with finer regions in location of the swirling flow (Figs. 6, 7). Maximum wall $\mathrm{y}^{+}$values were around 130 , maximum aspect ratio 12. Turbulence was modelled using Reynolds stress model to include the effects of turbulence anisotropy in strongly swirling flows.

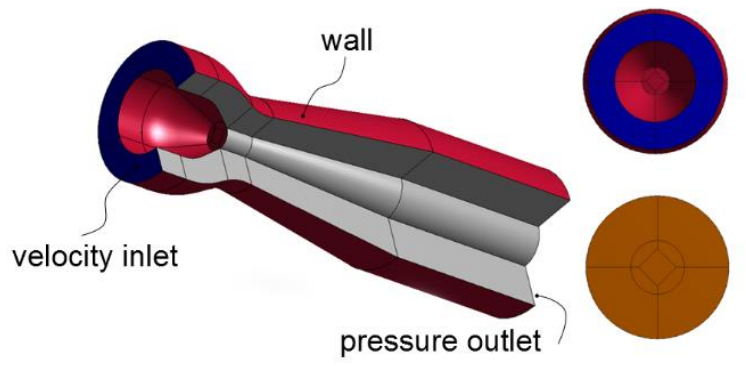

Fig. 6. Boundary conditions and decomposition of the flow domain

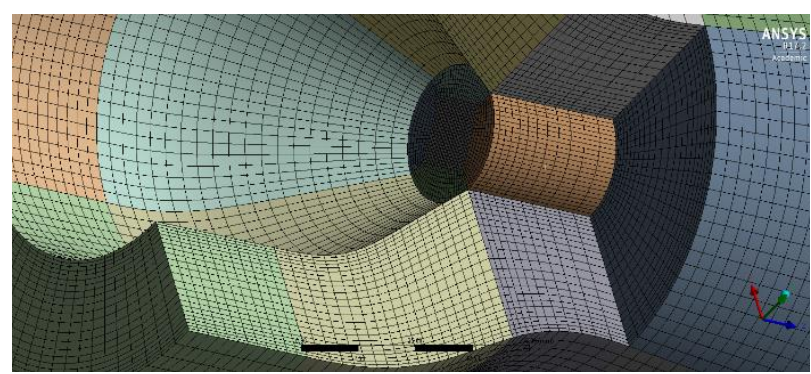

Fig. 7. Computational mesh close to the swirl generator cone
Resulting vortex rope has shape similar to the one observed during experimental testing (compare Fig. 5 and Fig.8). The dominant frequency connected with vortex rope rotation is $14.57 \mathrm{~Hz}$, which is very close to the experimental one $(14.95 \mathrm{~Hz})$. Amplitude of the first harmonic in position MG1 is underestimated by $20 \%$. This fact is quite common for transient flow simulations. Modeling also captured the plunging mode on lower frequency (about one third of the first harmonics) and with about $80 \%$ of the amplitude of the first harmonics (overestimation compared to experimental results).

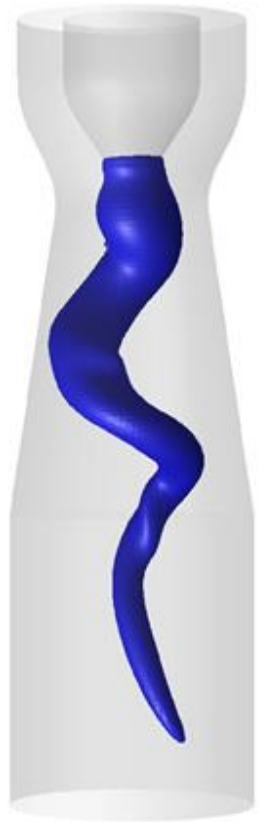

Fig. 8. Snapshot of the instantaneous pressure field visualizing the pressure contour of the vortex rope

Vortex rope onset is connected with instability of the shear layer in between two opposing streams under conditions of adverse pressure gradient (similar to KelvinHelmholtz instability). Therefore it was assumed that manipulation of the shear layer in location of highest strain rate would be most sensitive and provide best results. Magnitude of the strain rate tensor was computed in region according to Fig. 9. Strain rate tensor is defined by equation (1).

$$
v_{i j}=\frac{1}{2}\left(\frac{\partial v_{i}}{\partial x_{j}}+\frac{\partial v_{j}}{\partial x_{i}}\right)
$$

And its magnitude according to equation (2).

$$
\left\|v_{i j}\right\|=\sqrt{v_{i j} v_{i j}}
$$

Distribution of the strain rate tensor magnitude over the investigated region is plotted in figure 10 . 


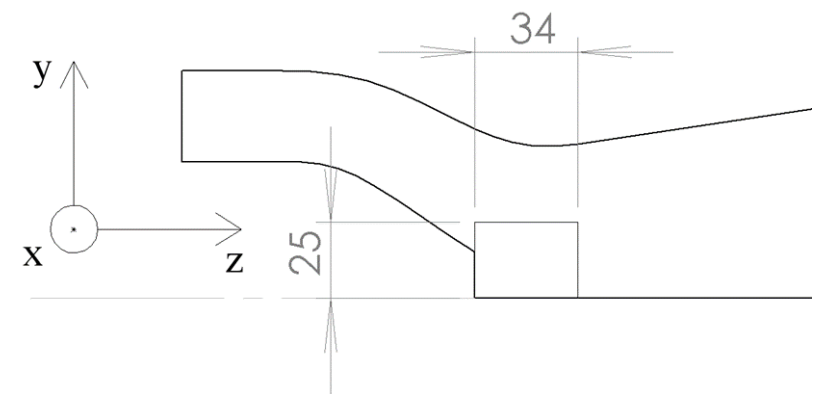

Fig. 9. Region for strain rate tensor computation

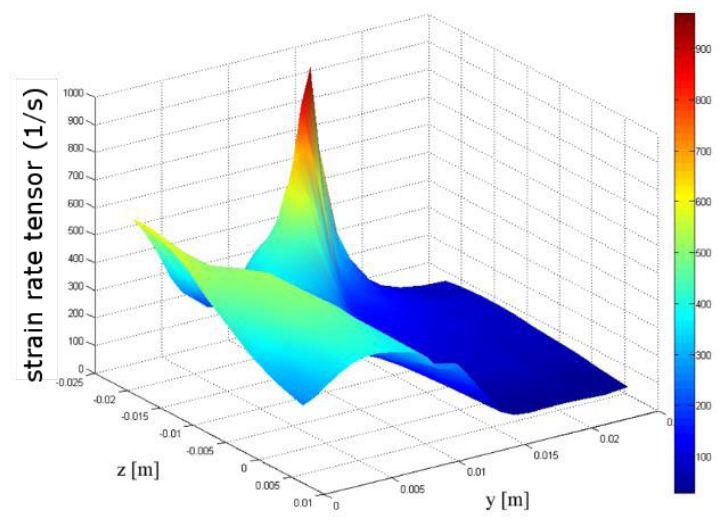

Fig. 10. Strain rate tensor magnitude

The highest value and hence the most sensitive location is placed just at the end of the conical region on the outer diameter of the circular hole, which was used by Susan-Resiga for water injection. This conclusion was later used to investigate different prospective types of water injection control. Their summary is provided in indicative figure 11 . a)

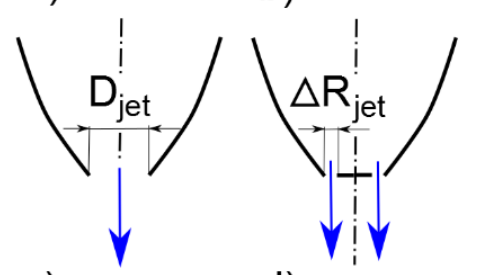

c)

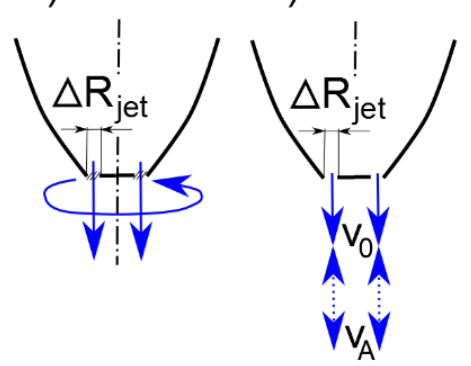

Fig. 11. Different types of water injection: a)full circular injection (Susan-Resiga [3, 4]), b) annular injection, c) annular injection with opposite swirl, d) annular injection with pulsating jet

\subsection{Different methods of water injection control} [6]

\subsubsection{Full circular jet control with $D_{\text {jet }}=30.6 \mathrm{~mm}$}

Water injection through full circular cross-section (see Fig.11a), which corresponds to the one applied by SusanResiga. It was used as a benchmark for comparison with other methods.

\subsubsection{Full circular jet control with $D_{\text {jet }}=20.6 \mathrm{~mm}$}

Similar to previous case, injection along the diffuser axis (see Fig.11a). Diameter for the injection is reduced to $67.3 \%$, flow area reduction is to $45.6 \%$. The jet is continuous in time.

\subsubsection{Annular jet with gap $\left(\Delta R_{\text {jet }}=2 \mathrm{~mm}\right)$}

Annular gap is designed at the edge of the conical region in the location, where highest magnitude of strain rate tensor was identified (see Fig.11b). Width of the gap is only $2 \mathrm{~mm}$. jet is continuous in time.

\subsubsection{Annular jet with gap $\left(\Delta R_{j e t}=5 \mathrm{~mm}\right)$}

Similar to previous case, but annular gap is $5 \mathrm{~mm}$ (see Fig. 11b). Jet is continuous in time.

\subsubsection{Annular jet $\left(\Delta R_{\text {jet }}=2 \mathrm{~mm}\right)$ with gap producing opposite swirl}

Stator blade cascade producing swirl with velocity angle of 45 degrees with axis and opposite sense of rotation than the vortex rope is placed in the annular gap of $2 \mathrm{~mm}$, see Fig. 11c. Hydraulic loss in the blade cascade is superimposed to the energy consumption.

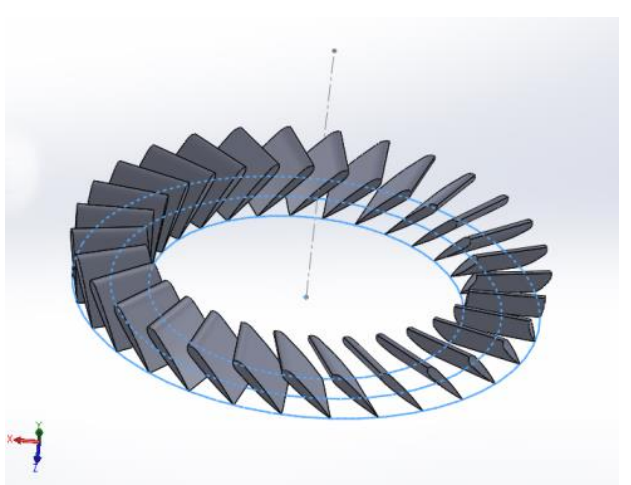

Fig. 12. Stator blade cascade in the annular gap

\subsubsection{Annular gap $\left(\Delta R_{\text {jet }}=2 \mathrm{~mm}\right)$ with pulsating jet}

To reduce the discharge consumption a pulsating jet was designed with constant stationary velocity component and 
with a pulsating one, whose frequency was modulated. Jet was emitting from annular gap of $2 \mathrm{~mm}$, see Fig. $11 \mathrm{~d}$.

$$
v(t)=v_{0}+v_{A} \sin (\omega t)
$$

Constant stationary value $v_{0}$ corresponding to discharge 3 $1 / \mathrm{s} \quad\left(v_{0}=v_{A}=14.5 \mathrm{~m} . \mathrm{s}^{-1}\right)$ was selected and pulsation frequency ranged from 0 to $29 \mathrm{~Hz}$.

\section{Results}

First, results were evaluated in terms of reduction of static pressure pulsations in location MG1 (Fig. 13). From the plot over the range of relative discharges $Q_{j e t} / Q$ from 0 to 0.5 it is clear that the best results are obtained with the original full circular jet. Acceptable results are also achieved using annular jet with opposite swirl and annular jet with 5 mm gap.

Best results in terms of energy consumption are again provided by the original full circular gap jet. Energy consumption increases with square of velocity.

It should be noted that pulsating jet did not disrupt the original flow field, but pulsations were superimposed to the pressure pulsations induced by vortex rope. Therefore pressure amplitudes have always been higher than the pulsations of the rope itself over whole range of the modulating frequencies. Results with pulsating jet are not plotted in Fig. 13, 14.

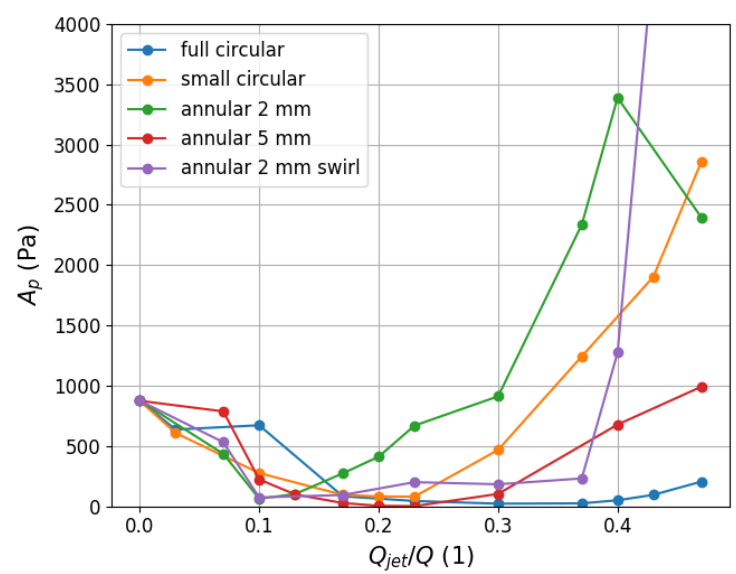

Fig. 13. Amplitudes of the static pressure in position MG1 for different methods of vortex rope mitigation

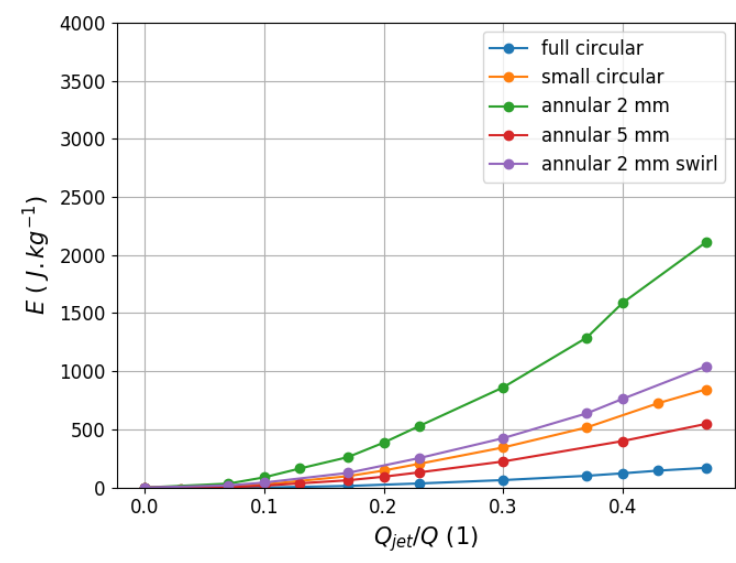

Fig. 14. Energy required for water jet supply for different methods of vortex rope mitigation

\section{Conclusion}

Numerical experiments with water jet injection for vortex rope pulsations mitigation were carried out. Altogether seven design modifications were tested. It was shown that none of them was better in pressure amplitudes suppression than the original one proposed by SusanResiga [3].

Manipulating the shear layer in location of highest strain rate did not prove to be a successful approach. Presented results indicate that vortex rope behaviour is especially influenced by flow along the diffuser axis in region of momentum deficit, where high amounts of the injected water provide best results. This finding is in agreement with recent observations of Goyal et al [7], who found out that the velocity profile in the decelerated region has to be influenced in appropriate way by bringing higher momentum of the fluid.

While the current design modifications did not generate better method than the original one, they provided important results for our future effort in the active control of the diffuser flow based on reduced order models built upon proper orthogonal decomposition of the swirling flow field [8], [9].

\section{Acknowledgements}

This research was supported by Czech Science Agency under project 17-01088S "3D instability of a shear layer in adverse pressure gradient" and Faculty of Mechanical Engineering, Brno University of Technology project FSI-S-17-4615.

\section{References}

1. A.T. Favrel, Dynamics of cavitation precessing vortex core for Francis turbines at part load operating conditions, PhD thesis, EPFL Lausanne, 2016

2. D. Štefan, P. Rudolf, Proper Orthogonal Decomposition of Pressure Fields in a Draft Tube Cone of the Francis (Tokke) Turbine Model. In Journal of Physics Conference Series. Journal of 
Physics: Conference Series, 2015. pp. 1-14, ISSN: 1742-6596.

3. R. Susan-Resiga, T.C. Vu, S. Muntean, G.D. Ciocan, B. Nennemann, Jet control of the draft tube vortex rope in Francis turbines at partial discharge, $23^{\text {rd }}$ IAHR Symposium, Yokohama, 2006

4. A.I. Bosioc, C. Tanasa, S. Muntean, R. Susan-Resiga, Unsteady pressure measurements and numerical investigation of jet control method in a conical diffuser with swirling flow, IOP Conference Series: Earth and Environmental Science, 12 (1), 2010

5. O. Petit, A.I. Bosioc, H. Nilsson, S. Muntean, R. Susan-Resiga, Unsteady simulations of the flow in a swirl generator using OpenFOAM, Int. Journal of Fluid Machinery and Systems, 4 (1), 2011, pp. 199208

6. J. Litera, Flow control in hydraulic turbine draft tube, diploma thesis, Brno University of Technology, 2017

7. R. Goyal, B.K. Gandhi, M.J. Cervantes, Experimental study of mitigation of a spiral vortex at high Reynolds number under an adverse pressure gradient, Physics of fluids, 29 (10), 2017

8. D. Štefan, P. Rudolf, S. Muntean, R. Susan-Resiga, Proper Orthogonal Decomposition of Self- Induced Instabilities in Decelerated Swirling Flows and Their Mitigation Through Axial Water Injection. Journal of Fluids Engineering - Transictions of the ASME, 2017, 139 (8), pp. 1-25. ISSN: 0098-2202

9. P. Rudolf, D. Štefan, R. Klas, Spatio- Temporal Description of the Swirling Flow in Hydraulic Turbine, Wasserwirtschaft, 1, 2015, pp. 18-22, ISSN: 0043- 0978 